Main Library

Strickhofstrasse 39

CH-8057 Zurich

www.zora.uzh.ch

\title{
Kristallstruktur eines Tetrahydro-1(1H)-isoindolons
}

Prewo, Roland ; Bieri, Jost H ; Widmer, Ulrich ; Heimgartner, Heinz

Abstract: Crystal Structure of a Tetrahydro-1(1H)-isoindolone. The structure of the tetrahydro-1(1H)isoindolone 4a, the product of an intra- molecular Diels-Alder addition, has been determined by X-ray analysis. The revised relative configuration of 4 a suggests a formation via the exo-transition state of the $[4+2]$-cycloaddition, followed by enolization to the more stable cis-fused product.

DOI: https://doi.org/10.1002/hlca.19810640527

Posted at the Zurich Open Repository and Archive, University of Zurich ZORA URL: https://doi.org/10.5167/uzh-97547 Journal Article

Originally published at:

Prewo, Roland; Bieri, Jost H; Widmer, Ulrich; Heimgartner, Heinz (1981). Kristallstruktur eines Tetrahydro-1(1H)-isoindolons. Helvetica Chimica Acta, 64(5):1515-1521.

DOI: https://doi.org/10.1002/hlca.19810640527 


\section{Kristallstruktur eines Tetrahydro-1 $(1 \mathrm{H})$-isoindolons}

von Roland Prewo, Jost H. Bieri, Ulrich Widmer ${ }^{\mathrm{I}}$ ) und Heinz Heimgartner ${ }^{2}$ )

Organisch-chemisches Institut der Universität Zürich, Winterthurerstrasse 190, CH-8057 Zürich

Herrn Prof. Dr. Conrad Hans Eugster zum 60. Geburtstag gewidmet

(14.V.81)

\section{Crystal Structure of a Tetrahydro-1 $(1 \mathrm{H})$-isoindolone}

\section{Summary}

The structure of the tetrahydro- $1(1 \mathrm{H})$-isoindolone $4 \mathrm{a}$, the product of an intramolecular Diels-Alder addition, has been determined by X-ray analysis. The revised relative configuration of $4 \mathrm{a}$ suggests a formation via the exo-transition state of the $[4+2]$-cycloaddition, followed by enolization to the more stable cis-fused product.

Vor einiger Zeit berichteten wir [1], dass die Umsetzung des 3-Dimethylamino2,2-dimethyl-2 $\mathrm{H}$-azirins (1) mit Sorbinsäurechlorid (2) das Acylamidinderivat 3 als (4:1)-Gemisch des $\left(2^{\prime} E, 4^{\prime} E\right)$ - und des $\left(2^{\prime} E, 4^{\prime} Z\right)$-Isomeren liefert (Schema 1). Die Thermolyse von 3 bei $170^{\circ}$ in Acetonitril (Bombenrohr) führt via intramolekulare Diels-Alder-Reaktion in 78\% Ausbeute zum 3a,4,5,7a-Tetrahydro-1 $(1 \mathrm{H})$-isoindolon 4 (Schema 1).

Schema 1
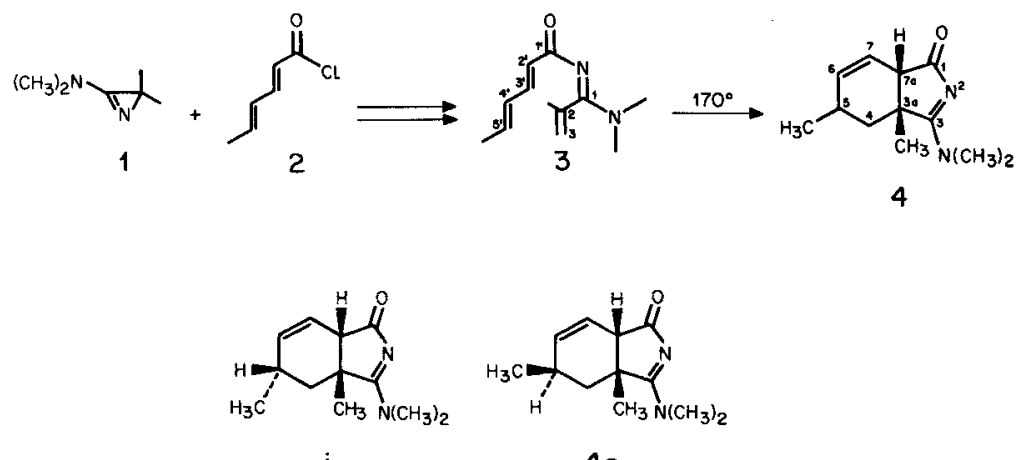

$4 a$

1) Pharmazeutische Forschungsabteilung der F. Hoffmann-La Roche \& Co. AG, CH-4002 Basel.

2) Korrespondenzautor. 
Die in [1] angegebene relative Konfiguration i des Cycloadduktes 4 wurde mittels 'H-NMR.-Spektroskopie hergeleitet; für die cis-Verknüpfung der beiden Ringe sprach die thermische Stabilität der Verbindung(vgl. die in [1] zit. Lit.). Die cis-Stellung der beiden $\mathrm{H}$-Atome an $\mathrm{C}(5)$ und $\mathrm{C}(7 \mathrm{a})$ und damit die trans-Stellung der beiden Methylgruppen an $\mathrm{C}(3 \mathrm{a})$ und $\mathrm{C}(5)$ wurde damals aufgrund der grossen homoallylischen Kopplung von $2,4 \mathrm{~Hz}$ postuliert, welche eine parallele Anordnung der beiden $\mathrm{C}, \mathrm{H}$-Bindungen an $\mathrm{C}(5)$ und $\mathrm{C}$ (7a) nahelegt.

Eine syn-koplanare Lage von $\mathrm{H}-\mathrm{C}(5)$ und $\mathrm{H}-\mathrm{C}(7 \mathrm{a})$ ist nur im einen der beiden cis-verknüpften Isomeren möglich, nämlich in $\mathbf{i}$; in Figur $l$ ist die aufgrund von Betrachtungen an Dreidingmodellen günstigste Konformation von i wiedergegeben. Dabei liegt der Cyclohexenring in einer «Pseudowannen-Konformation» vor.

Die Konformation des Isomeren $\mathbf{4 a}$, die auch eine grosse homoallylische Kopplung zwischen $\mathrm{H}-\mathrm{C}(5)$ und $\mathrm{H}-\mathrm{C}(7 \mathrm{a})$ erklären würde, entspricht etwa einer «Envelope-Konformation» und ist ebenfalls in Figur 1 dargestellt. Die relevanten $\mathrm{H}$-Atome liegen aber dabei nur nahezu anti-koplanar zueinander (der Winkel zwischen den Ebenen durch $\mathrm{H}-\mathrm{C}(5)-\mathrm{CH}_{3}$ und $\mathrm{H}-\mathrm{C}(7 \mathrm{a})-\mathrm{C}(1)$ beträgt etwa $20^{\circ}$ ). Diese Modellbetrachtungen führten dazu, dass wir damals - zur Erklärung der grossen homoallylischen Kopplung im ${ }^{1}$ H-NMR.-Spektrum - dem isolierten Tetrahydro-1 $(1 H)$-isoindolon die relative Konfiguration i zugeschrieben haben [1].

Wie wir schon in [1] erwähnt haben, fällt es schwer, die bevorzugte Bildung eines Stereoisomeren anhand von Modellbetrachtungen des Übergangszustandes der intramolekularen Diels-Alder-Reaktion zu erklären. Die in einem neueren Übersichtsartikel [2] zusammengestellten Beispiele von intramolekularen Diels-AlderReaktionen zeigen zudem, dass schon geringe strukturelle Unterschiede in der
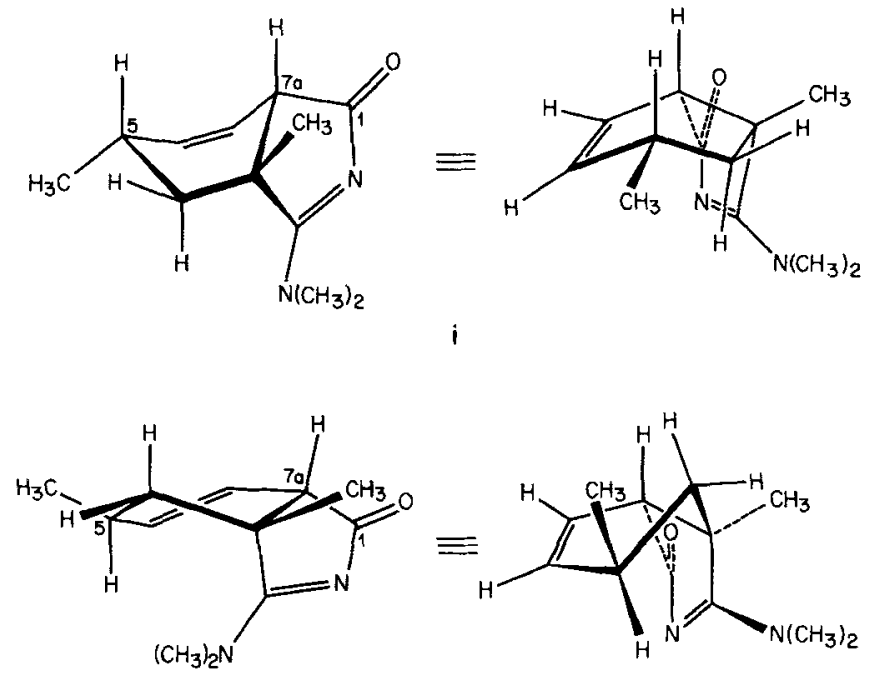

40

Fig. 1. Günstigste Konformationen der isomeren Tetrahydro-1(/H)-isoindolone $\mathbf{i}$ und $\mathbf{4 a}$ mit ungefähr paralleler Anordnung von $\mathrm{H}-\mathrm{C}(5)$ und $\mathrm{H}-\mathrm{C}(7 \mathrm{a})$ 
Brücke zwischen Dien und Dienophil das Verhältnis von exo-/endo-Addition stark beeinflussen können (vgl. dazu auch [3]).

Infolge der erwähnten Unsicherheiten bei der Strukturbestimmung des bei der Thermolyse des Acylamidinderivates 3 gebildeten 3a,4,5,7a-Tetrahydro-1 $(1 \mathrm{H})$ isoindolons 4 vom Smp. 123,5-124,5 (Äther/Aceton) haben wir nachträglich eine Röntgen-Kristallstrukturanalyse durchgeführt.

Kristalldaten. Farblose Kristalle, Formel $\mathrm{C}_{12} \mathrm{H}_{18} \mathrm{~N}_{2} \mathrm{O}$, Mol-Gew. 206,29; Kristallsystem orthorhombisch, Raumgruppe $P$ bca; Gitterparameter (bei $-140^{\circ}$ ): $a=10,884 \pm 0,008, b=12,244 \pm 0,007$, $c=17,037 \pm 0,008 \AA ; V=2270,4 \AA^{3}, Z=8$.

Intensitätsmessungen und Strukturbestimmung. Die Intensitäten wurden mit einem Nicolet R3Vierkreisautodiffraktometer mit Tieftemperaturzusatz LT-1 bei etwa $-140^{\circ}$ mit $M o K_{\alpha}$-Strahlung im $\omega$-Scan gemessen; Messbereich (20) $0-50^{\circ}$. Von insgesamt 1994 symmetrieunabhängigen Reflexen wurden $1446(72,5 \%)$ als beobachtet angenommen $(I \geqslant 2,5 \sigma(I))$ und zur Strukturbestimmung verwendet. Für die Strukturbestimmung mit direkten Methoden und die Verfeinerung bis zu einem R-Wert von 0,045 wurde das Programmsystem SHELXTL [4] verwendet. Alle H-Atome wurden durch DifferenzFourieranalysen lokalisiert. Ihre Verfeinerung erfolgte mit individuellen, isotropen Temperaturfaktoren, während die schwereren Atome anisotrop verfeinert wurden. Resultate: Siehe Tabellen 1 und 2 sowie Figuren 2 und $3^{3}$ ).

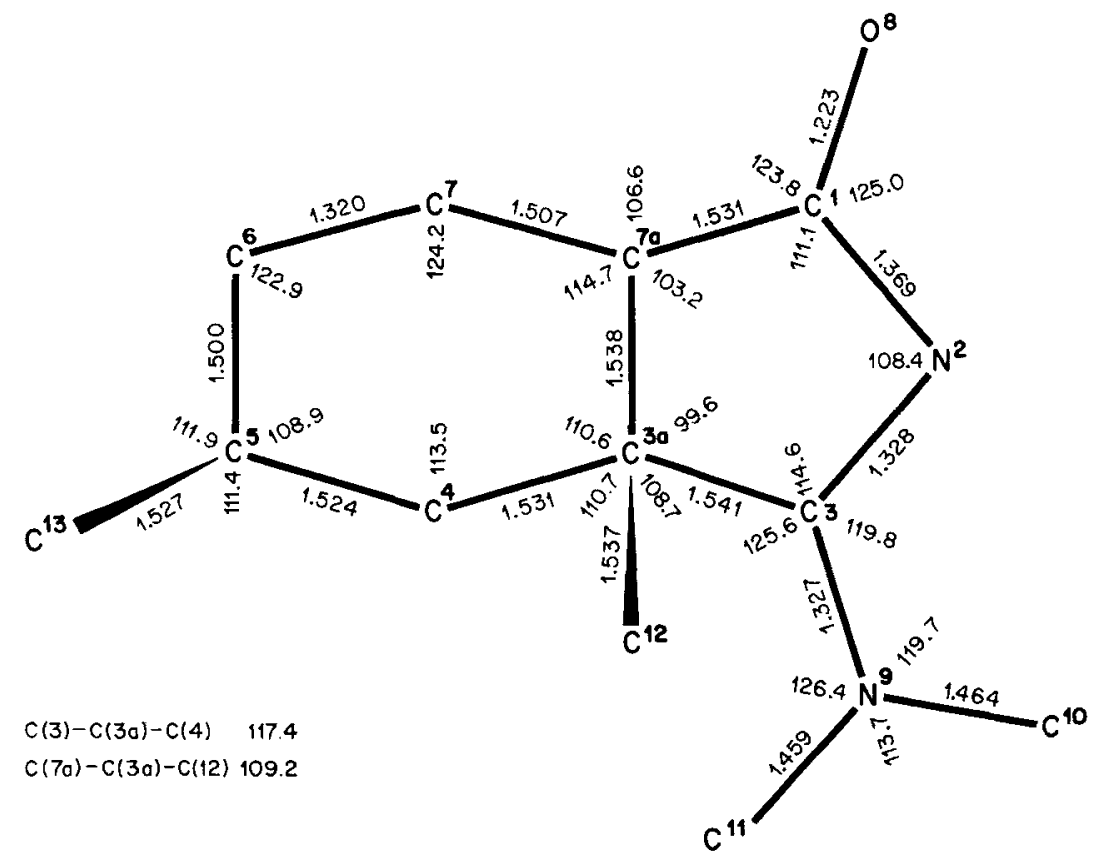

Fig.2. Struktur des 3-Dimethylamino-3a,5-dimethyl-3a,4,5,7a-tetrahydro-1 $(1 \mathrm{H})$-isoindolons (4a) mit verwendeter Numerierung der Atome, Bindungslängen $(A$, Standardabweichungen 0,003 $A)$ und Bindungswinkeln (Grad, Standardabweichungen $0,2^{\circ}$ )

3) Weitere Angaben zur Kristallstruktur von 4a können bei J.H.B. eingeholt werden. 
Tabelle 1. Koordinaten der Atome (Standardabweichungen $\times 10000$ ) von 4a und isotrope Temperaturfaktoren

\begin{tabular}{|c|c|c|c|c|}
\hline Atom & $\mathrm{X}$ & $\mathrm{Y}$ & Z & $\mathrm{U}$ \\
\hline$C(1)$ & $0,2055(2)$ & $0,3964(2)$ & $0,3725(1)$ & a) \\
\hline$N(2)$ & $0.2489(2)$ & $0,3145(1)$ & $0,4197(1)$ & a) \\
\hline$C(3)$ & $0,3624(2)$ & $0,2900(2)$ & $0,3971(1)$ & a) \\
\hline$C(3 a)$ & $0,4066(2)$ & $0,3518(2)$ & $0,3235(1)$ & a) \\
\hline $\mathrm{C}(4)$ & $0,5347(2)$ & $0,4034(2)$ & $0,3265(1)$ & a) \\
\hline$C(5)$ & $0,5506(2)$ & $0,4850(2)$ & 0,3933 (1) & a) \\
\hline$c(6)$ & $0,4496(2)$ & $0,5681(2)$ & $0,3888(1)$ & a) \\
\hline$C(7)$ & $0,3446(2)$ & $0,5498(2)$ & $0,3524(1)$ & a) \\
\hline$C(7 a)$ & $0,3070(2)$ & $0,4408(2)$ & $0,3191(1)$ & a) \\
\hline$O(8)$ & $0,1010(1)$ & $0,4330(1)$ & $0,3744(1)$ & a) \\
\hline$N(9)$ & $0,4254(2)$ & $0,2140(1)$ & $0,4352(1)$ & a) \\
\hline$C(10)$ & $0,3710(2)$ & $0,1607(2)$ & $0,5039(1)$ & a) \\
\hline$C(11)$ & $0.5441(2)$ & $0,1687(2)$ & $0,4123(1)$ & a) \\
\hline$C(12)$ & $0,3946(2)$ & $0,2759(2)$ & $0,2521(1)$ & a) \\
\hline$C(13)$ & $0,6776(2)$ & $0,5381(2)$ & $0,3912(1)$ & a) \\
\hline$H(1)-C(4)$ & $0,5410(14)$ & $0,4425(12)$ & $0,2771(1 !)$ & 0,025 \\
\hline $\mathrm{H}(2)-\mathrm{C}(4)$ & $0,6004(14)$ & $0,3479(13)$ & $0.3276(10)$ & 0,019 \\
\hline$H-C(5)$ & $0,5455(14)$ & $0,4476(13)$ & $0.4445(12)$ & 0,029 \\
\hline $\mathrm{H}-\mathrm{C}(6)$ & $0,4648(14)$ & $0,6345(14)$ & $0.4143(11)$ & 0,029 \\
\hline $\mathrm{H}-\mathrm{C}(7)$ & $0,2842(14)$ & $0,6063(14)$ & $0,3499(11)$ & 0,030 \\
\hline $\mathrm{H}-\mathrm{C}(7 \mathrm{a})$ & $0,2783(14)$ & $0,4489(13)$ & $0,2638(11)$ & 0,031 \\
\hline$H(1)-C(10)$ & $0,4378(16)$ & $0,1432(15)$ & $0,5438(13)$ & 0,056 \\
\hline $\mathrm{H}(2)-\mathrm{C}(10)$ & $0,3346(16)$ & $0,0934(15)$ & $0,4864(13)$ & 0,059 \\
\hline$H(3)-C(10)$ & $0,3081(15)$ & $0,2070(14)$ & $0,5250(13)$ & 0,056 \\
\hline $\mathrm{H}(1)-\mathrm{C}(11)$ & $0,5656(15)$ & $0,1928(14)$ & $0,3625(12)$ & 0,040 \\
\hline $\mathrm{H}(2)-\mathrm{C}(11)$ & $0,6092(15)$ & $0,1928(14)$ & $0,4522(13)$ & 0,054 \\
\hline $\mathrm{H}(3)-\mathrm{C}(11)$ & $0,5307(16)$ & $0,0847(14)$ & $0,4096(13)$ & 0,058 \\
\hline $\mathrm{H}(1)-\mathrm{C}(12)$ & $0,4183(14)$ & $0,3197(13)$ & $0,2066(12)$ & 0,030 \\
\hline $\mathrm{H}(2)-\mathrm{C}(12)$ & $0,4480(14)$ & $0,2092(14)$ & $0,2548(12)$ & 0,035 \\
\hline $\mathrm{H}(3)-\mathrm{C}(12)$ & $0,3046(14)$ & 0,2497 (14) & $0,2490(12)$ & 0,034 \\
\hline $\mathrm{H}(1)-\mathrm{C}(13)$ & $0,6858(14)$ & $0,5800(13)$ & $0,3411(11)$ & 0,026 \\
\hline $\mathrm{H}(2)-\mathrm{C}(13)$ & $0,7438(16)$ & $0,4826(15)$ & $0,3896(12)$ & 0,042 \\
\hline $\mathrm{H}(3)-\mathrm{C}(13)$ & $0,6954(16)$ & $0,5849(15)$ & $0.4349(14)$ & 0,061 \\
\hline
\end{tabular}

a) Anisotrope Temperaturfaktoren s. Tabelle 2.

Diskussion der Molekelstruktur. - Die Kristallstruktur 4a des Thermolyseproduktes von 3 ist durch die Koordinaten der Atome in Tabelle $l$ definiert; die für die Strukturbestimmung verwendete Atomnumerierung, die Bindungslängen und Bindungswinkel sind in Figur 2 wiedergegeben. Die räumliche Struktur der Molekel ist aus der Stereoprojektion (Fig. 3) ersichtlich. Daraus geht deutlich hervor, dass die beiden Ringe cis-verknüpft sind und sich die Methylgruppen an C(3a) und $\mathrm{C}(5)$ ebenfalls in cis-Stellung befinden, wobei beide eine pseudoäquatoriale Lage im Cyclohexenring einnehmen. Die im Kristall vorliegende Konformation der Molekel entspricht der in Figur 1 für $\mathbf{4 a}$ angegebenen; eine ähnliche Konformation muss aufgrund der grossen homoallylischen Kopplung im ${ }^{1}$ H-NMR.Spektrum auch in Lösung populiert sein. Das $N$-Acylamidinsystem $(\mathrm{O}(8), \mathrm{C}(1)$, $\mathrm{N}(2), \mathrm{C}(3), \mathrm{N}(9))$ ist nahezu planar, was aus den folgenden Torsionswinkeln hervorgeht: $\mathrm{O}(8)-[\mathrm{C}(1)-\mathrm{N}(2)]-\mathrm{C}(3) \quad 175,0^{\circ}, \quad \mathrm{C}(1)-[\mathrm{N}(2)-\mathrm{C}(3)]-\mathrm{N}(9) \quad 178,6^{\circ}$, 
Tabelle 2. Anisotrope Temperaturfaktoren

$\mathrm{T}=\exp \left[-2 \pi^{2}\left(\mathrm{U}_{11} \mathrm{~h}^{2} \mathrm{a}^{* 2}+\mathrm{U}_{22} \mathrm{k}^{2} \mathrm{~b}^{* 2}+\mathrm{U}_{33} \mathrm{l}^{2} \mathrm{c}^{* 2}+2 \mathrm{U}_{12} \mathrm{hka}^{*} \mathrm{~b}^{*}+2 \mathrm{U}_{13} \mathrm{hla}^{*} \mathrm{c}^{*}+2 \mathrm{U}_{23} \mathrm{klb}^{*} \mathrm{c}^{*}\right)\right]$

\begin{tabular}{llllrrr}
\hline Atom & $\mathrm{U}_{11}$ & $\mathrm{U}_{22}$ & $\mathrm{U}_{33}$ & \multicolumn{1}{c}{$\mathrm{U}_{23}$} & \multicolumn{1}{c}{$\mathrm{U}_{13}$} & \multicolumn{1}{c}{$\mathrm{U}_{12}$} \\
\hline $\mathrm{C}(1)$ & 0,018 & 0,023 & 0,021 & $-0,005$ & 0,000 & 0,001 \\
$\mathrm{~N}(2)$ & 0,018 & 0,024 & 0,021 & 0,000 & 0,002 & 0,001 \\
$\mathrm{C}(3)$ & 0,019 & 0,017 & 0,021 & $-0,004$ & $-0,001$ & $-0,004$ \\
$\mathrm{C}(3 \mathrm{a})$ & 0,020 & 0,020 & 0,018 & $-0,004$ & 0,001 & 0,000 \\
$\mathrm{C}(4)$ & 0,018 & 0,024 & 0,020 & $-0,001$ & 0,003 & 0,000 \\
$\mathrm{C}(5)$ & 0,029 & 0,024 & 0,018 & 0,002 & 0,001 & $-0,005$ \\
$\mathrm{C}(6)$ & 0,035 & 0,019 & 0,028 & $-0,003$ & 0,008 & $-0,005$ \\
$\mathrm{C}(7)$ & 0,030 & 0,018 & 0,030 & 0,004 & 0,007 & 0,004 \\
$\mathrm{C}(7 \mathrm{a})$ & 0,021 & 0,028 & 0,016 & 0,003 & $-0,001$ & 0,002 \\
$\mathrm{O}(8)$ & 0,020 & 0,036 & 0,034 & 0,001 & 0,001 & 0,005 \\
$\mathrm{~N}(9)$ & 0,021 & 0,020 & 0,030 & 0,004 & $-0,002$ & 0,001 \\
$\mathrm{C}(10)$ & 0,036 & 0,030 & 0,028 & 0,007 & $-0,002$ & $-0,002$ \\
$\mathrm{C}(11)$ & 0,022 & 0,026 & 0,043 & 0,003 & $-0,001$ & 0,003 \\
$\mathrm{C}(12)$ & 0,031 & 0,031 & 0,024 & $-0,009$ & 0,002 & $-0,004$ \\
$\mathrm{C}(13)$ & 0,031 & 0,031 & 0,032 & 0,001 & $-0,006$ & $-0,009$ \\
\hline
\end{tabular}

$N(2)-[C(3)-N(9)]-C(10)-2,4^{\circ}$ und $N(2)-[C(3)-N(9)]-C(11) 172,2^{\circ}$. Diese Planarität zeigt sich auch in der ausgeprägten Konjugation der $\pi$-Systeme, die zu den sehr kurzen (formalen) C, N-Einfachbindungen $C(1)-N(2)$ von 1,369 $\AA$ und $\mathrm{C}(3)-\mathrm{N}$ (9) von $1,327 \AA$ führt, während die N (2), C (3)-Doppelbindung mit 1,328 $\AA$ relativ lang ist (Soll-Werte: $\mathrm{C}-\mathrm{N} 1,47 \AA$ und $\mathrm{C}=\mathrm{N} 1,30 \AA)^{4}$ ). Der deutliche Amidinium-Charakter von $\mathrm{C}(3)$ spiegelt sich in dessen chemischer Verschiebung von $186,5 \mathrm{ppm}$ im ${ }^{13} \mathrm{C}$-NMR.-Spektrum $\left(\mathrm{CDCl}_{3}\right)$ wider $\left.(\operatorname{vgl} \text {. dazu auch [8] })^{5}\right)$.
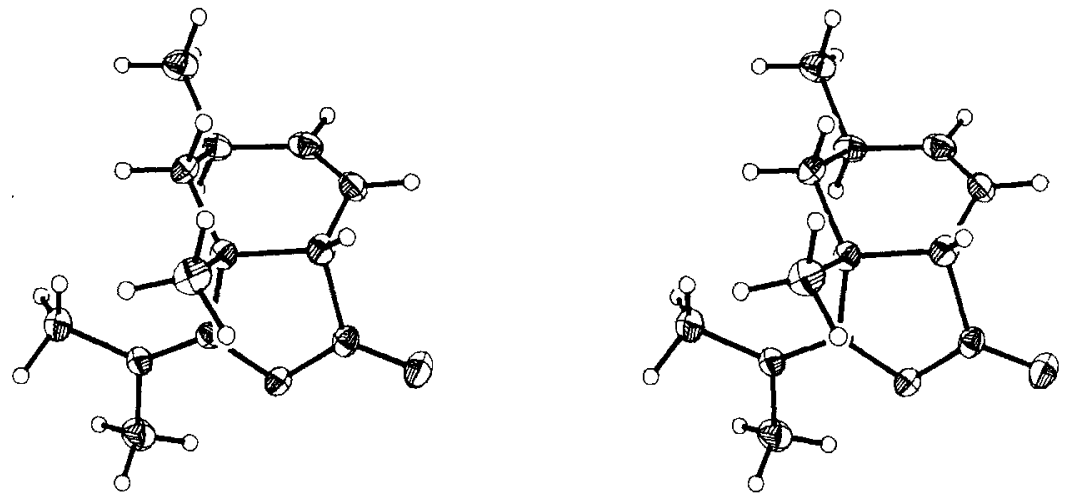

Fig. 3. Stereoprojektion der Molekel 4a

4) Ähnliche Bindungslängen sind für das Acylamidinsystem in 2-Anilino-5-phenyl-2-thiazolin-4-on bestimmt worden [5], während in einem cyclischen, 7-gliedrigen Acylamidin-Derivat die Unterschiede zwischen $\mathrm{C}, \mathrm{N}$-Doppel- und $\mathrm{C}, \mathrm{N}$-Einfachbindung viel deutlicher sind [6]. Die Amidingruppierung in einem 2-Amino-1-pyrrolin-Derivat weist für die formale C,N-Doppel- und C,NEinfachbindung Längen von 1,31 bzw. 1,32 A auf [7].

5) Die Zuordnung der beiden Signale bei tiefem Feld (189,4 ppm (C(1)) und 186,5 ppm (C(3)) [1]) erfolgte mit Hilfe der nichtentkoppelten Spektren. Dabei erscheint das Signal bei 189,4 ppm als relativ scharfes Dublett, während dasjenige bei 186,5 ppm als schmales Multiplett auftritt. Herrn Dr. W. Schwotzer sei für die Aufnahme der Spektren gedankt. 
Aufgrund der durch die Röntgen-Kristallstrukturanalyse bewiesenen relativen Konfiguration 4a des 3-Dimethylamino-3a,5-dimethyl-3a,4,5,7a-tetrahydro1 (1 H)-isoindolons scheint die intramolekulare Diels-Alder-Reaktion von 3 (Schema 2) via den Übergangszustand exo-3 zum gespannten, trans-verknüpften $\mathbf{4 b}$ zu verlaufen, welches durch eine Enolisierung in das thermodynamisch stabilere Isomere 4 a übergeht.

Am ehesten vergleichbar mit der hier beschriebenen intramolekularen DielsAlder-Reaktion ist diejenige von Sorbinsäure-diallylamid [9]. Bei dessen Erhitzung auf $156^{\circ}$ in DMF entsteht ein ca. (1:1)-Gemisch des trans- und des cis-verknüpften 2,3,3a, 4, 5, 7a-Hexahydro-1 $(1 H)$-isoindolons, die über den exo-bzw. den endoÜbergangszustand $\left.{ }^{6}\right)$ gebildet werden. Im Gegensatz dazu liefert $N$-(1,3-Butadienyl)- $N$-(3-butenyl)-methylurethan bei $160^{\circ}$ nur das cis-Hexahydroindolderivat [10]. Die hier beobachtete Änderung der Stereoselektivität der Cycloaddition beim Übergang von einem Substrat, bei welchem die Amidcarbonylgruppe in der Brücke zwischen Dien und Dienophil integriert ist, zu einem solchen mit externer Amidcarbonylgruppe ist auch für die Reaktion von $N$-(Buta-1,3-dienyl)-amiden beschrieben worden [10]. In diesem Fall kann die Stereoselektivität mit der Koplanarität von Amidfunktion und Diensystem im exo-bzw. endo-Übergangszustand erklärt werden. Bevorzugt über den zu trans-verknüpften Hexahydro-1-isoindolonen führenden exo-Übergangszustand verlaufen die intramolekularen Diels-AlderReaktionen von Pentadienylacrylamiden [11] [12], während das entsprechende, carbonylgruppenfreie Allyl-pentadienylamin wiederum das cis-verknüpfte Isomere (via endo-Übergangszustand) als Hauptprodukt liefert [11]. Einen sehr grossen Einfluss auf die Energie des Übergangszustandes hat dabei offenbar ein terminales $\pi$-System am Dienophilteil: so ergibt $N$-(4,5-Diphenyl-2,4-pentadienyl)- $N$-methylacrylamid ein (1:1)-Gemisch von trans- und cis-verknüpftem Cycloaddukt [11], N-(4,5-Diphenyl-2,4-pentadienyl)- $N$-methyl-zimtsäureamid führt bei der Thermolyse zu einem (8:1)-Gemisch zugunsten des trans-Isomeren und im Falle der entsprechenden äthoxycarbonyl-substituierten Acrylamide wird nur das trans-Produkt gebildet. Diese Beobachtung wird mit der bevorzugten endo-Lage des terminalen $\pi$-Systems im exo-Übergangszustand erklärt [11] (vgl. auch [13]). Andererseits wird aus $N$-Benzyl- $N$-cinnamyl-zimtsäureamid in siedendem $o$-Dichlorbenzol $\left(180^{\circ}\right)$ nur das cis-Benz [f]isoindol-Derivat isoliert [14]; hier reagiert jedoch - im Gegensatz zu den vorangehend beschriebenen Beispielen - der Zimtsäure-Teil als Dienkomponente.

Schema 2

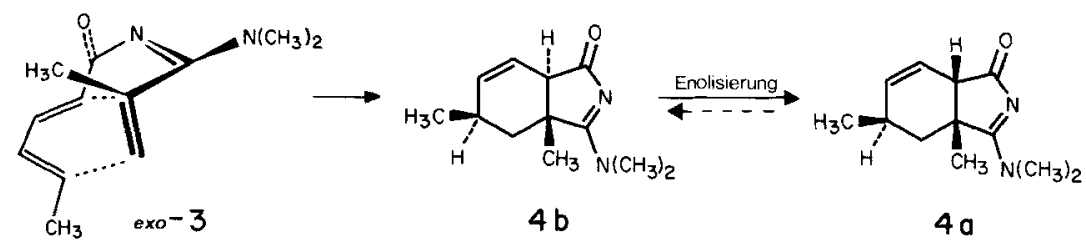

6) Die Bezeichnungen exo und endo für den Übergangszustand beziehen sich auf die Lage der Brücke zwischen Dien und Dienophil (vgl. auch [2]). 
Eine Bevorzugung des exo-Übergangszustandes unter Ausbildung von transOctahydronaphthalin-1-onen wird sowohl für die intramolekulare Diels-AlderReaktion von Deca-1, 7, 9-trien-1-on [15] und einem substituierten Deca-1,3,9-trien5-on [16] als auch von carbonylgruppen-freien Deca-1,3,9-trienen [17] beobachtet. In diesen Fällen sind möglicherweise sterische Effekte in den Übergangszuständen mit angenommener Sesselkonformation für die Begünstigung der exo-Addition verantwortlich.

Für die in Schema 2 postulierte, unter den Reaktionsbedingungen offenbar rasch verlaufende Isomerisierung vom kinetisch kontrollierten trans- zum thermodynamisch stabileren cis-Produkt via Enolisierung sind ebenfalls Analogiefälle bekannt. So erfolgt z. B. die Epimerisierung rein thermisch dann leicht, wenn das enolisierbare $a-\mathrm{H}$ zusätzlich von einer Doppelbindung flankiert ist [9] [16]. Im Falle von $\mathbf{4 b \rightarrow 4 a}$ (Schema 2) ist zudem eine Basenkatalyse durch das Amidinsystem nicht auszuschliessen.

Wir danken Herrn H. Rippstein, Scintag Inc., Sunnyvale CA, für seine Hilfe bei der Ermittlung der Röntgen-Kristallstruktur.

\section{LITERATURVERZEICHNIS}

[1] U. Widmer, H. Heimgartner \& H. Schmid, Helv. Chim. Acta 61, 815 (1978).

[2] G. Brieger \& J.N. Bennett. Chem. Rev. 80, 63 (1980).

[3] W. Oppolzer, Angew. Chem. 89, 10 (1977).

[4] G.M. Sheldrick, 'SHELXTL, An integrated system for solving, refining and displaying crystal structures from diffraction data, Revision 2.2, 1979', Universität Göttingen.

[5] R. Bally \& J.-P. Mornon, Acta Crystallogr. B29, 1157 (1973).

[6] B. Scholl, J. H. Bieri \& H. Heimgartner, Helv. Chim. Acta 61, 3050 (1978).

[7] K.-H. Pfoertner, W. Meister, W.E. Oberhänsli, P. Schönholzer \& W. Vetter, Helv. Chim. Acta 58, 846 (1975).

[8] J. Lukáć \& H. Heimgartner, Helv. Chim. Acta 62, 1236 (1979).

[9] G. Fráter, Tetrahedron Lett. 1976, 4517.

[10] W. Oppolzer \& W. Fröstl, Helv. Chim. Acta 58, 590 (1975).

[11] H.W. Gschwend, A.O. Lee \& H.-P. Meier, J. Org. Chem. 38, 2169 (1973),

[12] H.W. Gschwend \& H. -P. Meier, Angew. Chem. 84, 291 (1972).

[13] H. W. Gschwend, Helv. Chim. Acta 56, 1763 (1973).

[14] W. Oppolzer, R. Achini, E. Pfenninger \& H.P. Weber, Helv. Chim. Acta 59, 1186 (1976).

[15] W. Oppolzer \& R. L. Snowden, Tetrahedron Lett. 1976, 4187.

[16] F. Näf, R. Decorzant \& W. Thommen, Helv. Chim. Acta 62, 114 (1979).

[17] S. R. Wilson \& D.T. Mao, J. Am. Chem. Soc. 100, 6289 (1978). 